\title{
A importância do diagnóstico de enfermagem: visão dos enfermeiros
}

\author{
The important nursing diagnosis: vision of nurses \\ La importancia del diagnóstico de enfermería: visión de enfermeras
}

Lúcio Henrique D’avila Moreira

ORCID: https://orcid.org/0000-0002-6684-8333 Fundação Educacional do Município de Assis, Brasil E-mail: luciodavela@hotmail.com

Michelle Venâncio Hong

ORCID: https://orcid.org/0000-0002-4684-2196 Fundação Educacional do Município de Assis, Brasil

E-mail: venanaciomichelle_hong@hotmail.com

Daniel Augusto da Silva

ORCID: https://orcid.org/ 0000-0002-2716-6700 Fundação Educacional do Município de Assis, Brasil E-mail: daniel.augusto@unifesp.br

Rosângela Gonçalves da Silva

ORCID: https://orcid.org/0000-0002-3223-750X Fundação Educacional do Município de Assis, Brasil E-mail: roseziquinelli@gmail.com

\begin{abstract}
Resumo
Os diagnósticos assim como todas outras fases do processo de enfermagem desempenham papel fundamental para evolução do cliente, os mesmos norteiam todo planejamento da equipe de enfermagem sobre a implementação dos cuidados que atendam necessidades específicas de todos os pacientes no processo de saúde e doença. Este trabalho teve como objetivos levantar junto aos profissionais enfermeiros, o nível de conhecimento acerca dos diagnósticos de enfermagem e sua relação sob a ótica do processo de enfermagem e teorias de enfermagem. Tratou-se de uma pesquisa de campo com caráter qualitativo, tendo como sujeitos do estudo enfermeiros(as) ligados diretamente assistência, na atenção terciária de um hospital do interior paulista, que abordou os conhecimentos desses profissionais acerca da importância dos diagnósticos de enfermagem. Dos 18 (100\%) participantes, apenas $4(22,22 \%)$ responderam que se pautam na Teoria NHB de Wanda de Aguiar Horta e utilizam impressos eletrônicos da Taxonomia da Nanda-I. Os demais 14 (77,77\%), não souberam responder. A busca por um cuidado humanizado e uma profissão embasada cientificamente se faz necessário para toda á prática do cuidado, os diagnósticos de enfermagem sendo uma das fases do PE são essenciais para toda evolução dos pacientes, porém muitas vezes os diagnósticos de enfermagem não são aplicados de maneira correta, como foi possível constatar através dos dados e opiniões obtidos durante este estudo.
\end{abstract}

Palavras-chave: Diagnósticos de enfermagem; Assistência; Processo de enfermagem.

\begin{abstract}
Diagnoses, as well as all other phases of the nursing process (NP), play a fundamental role in the evolution of the client, they guide all planning of the nursing team on the implementation of care that meets the specific needs of all patients in the health and illness process. This work, had a objectives raise with the professional nurses, the level of knowledge about the nursing diagnoses and its relationship from the perspective of nursing process and nurging theories. It was a qualitative field research, having nurses as research subjects, directly linked to assistance, in tertiary care of a hospital in the interior of São Paulo, who addressed the knowledge of these professionals about the nursing diagnoses importance. The $18(100 \%)$ participants, only $4(22,22 \%)$ answered that they based on Theory NHB by Wanda de Aguiar Horta and use electronic printouts from Nanda-I Taxonomy. The others 14 (77,77\%), couldn't answer. The search for humanized care and a scientifically embased profession, it's necessary for all care practice, the nursing diagnoses that is the phases of NP are essential for all evolution of the patients, but many times the nursing diagnoses not applied correctly, how was it possible to verify with the data and opinion obtained in this study.
\end{abstract}

Keywords: Nursing diagnoses; Assistance; Nursing process.

\section{Resumen}

Los diagnósticos y todas las demás fases del proceso de enfermería (PE), desempeña un papel fundamental en la evolución del cliente, guían toda la planificación del equipo de enfermería en la implementación del cuidado que atención satisface las necesidades específicas de todos los pacientes en el proceso de salud y enfermedad. Este trabajo, tuvo como objetivo levantarse juntos con las enfermeras profesionales, el nível de conocimiento en diagnóstico de enfermería y la relación desde la perspectiva de proceso de enfermería y terorías de enfermería. Fue uma investigación 
de campo cualitativa, teniendo como sujetos de estúdio enfermeras directamente vinculado a la asistence, the la atención terciaria de un hospital en el interior de São Paulo, quien abordó el conocimiento de estos profesionales la importancia de diagnósticos de enfermería. De los 18 (100\%) participantes, solamente $4(22,22 \%)$, renpondió al uso basado en Teorías NHB de Wanda de Aguiar Horta y usa impressiones electrónicas de Taxonomía Nanda-I. Los demas 14 $(77,77 \%)$, no pudo responder. La búsqueda de cuidados humanizados y una profesión con base científica es necesaria para todas las prácticas de cuidado, diagnósticos de enfermería, siendo una de las fases de PE, son esenciales para toda la evolución del paciente, sin embargo muchas vezes los diagnósticos de enfermería no se aplican correctamente, como fue posible verificar a través de los datos y opiniones obtenidas durante este estudio.

Palabras clave: Diagnósticos de enfermería; Asistencia; Proceso de enfermería.

\section{Introdução}

As práticas de enfermagem desempenham papel fundamental no cuidado ao paciente, com passar dos anos ganhou-se novos conceitos e ferramentas que facilitassem as atividades do profissional de enfermagem. Entre elas o processo de enfermagem (PE) descrito e implantado por Wanda de Aguiar Horta em 1970, que foi divido em seis fases: Histórico de enfermagem, diagnóstico de enfermagem, plano assistencial, plano de cuidados ou prescrição de enfermagem, evolução e prognóstico (Horta, 2011).

No entanto, de acordo com a Resolução Cofen 358/2009 as fases atuais do processo de enfermagem são: coleta de dados, diagnóstico de enfermagem, planejamento, implementação e avaliação.

É essencial que todas essas etapas estejam em harmonia, onde temos a sistematização da assistência de Enfermagem, que sistematiza todas as fases do processo de enfermagem, tornando o serviço do profissional mais organizado e passível de acontecer, de modo a ser prestado de maneira humanizada e cientificamente respaldada (Cofen, 2009; Belfort et al., 2020; Morais, Brasil, Tolfo, Costa, \& Lohmann, 2020).

Os diagnósticos assim como todas outras fases do PE desempenham papel fundamental para evolução do cliente, os mesmos norteiam todo planejamento da equipe de enfermagem sobre a implementação dos cuidados que atendam necessidades específicas de todos os pacientes no processo de saúde e doença (Silva \& Moreira, 2020; Mazzo, 2013).

Braga e Cruz (2003) Afirmam que as intervenções de enfermagem devem estar fundamentas na avaliação do estado de saúde do indivíduo, diante disso é necessário que se adote o diagnóstico como referência.

Lepak (2015) define que o diagnóstico de enfermagem é o julgamento clínico diante uma resposta humana, de acordo com as condições do processo de saúde de vida, sendo este envolvido na sociedade, família, grupo ou comunidade.

Desta maneira, este trabalho buscou identificar, através dos profissionais de enfermagem, o nível de conhecimento acerca dos diagnósticos de enfermagem e sua relação sob a ótica do processo de enfermagem e teorias de enfermagem

\section{Metodologia}

Trata-se de uma pesquisa de campo de caráter qualitativo que avaliou o conhecimento dos enfermeiros sobre os diagnósticos de Enfermagem.

A amostra, de caráter não probabilístico aleatório por conveniência, foi composta por 18 enfermeiros, que se conseguiu abordar, e que atuam diretamente na assistência ao cliente/paciente, na atenção terciária, de um hospital do interior paulista.

As entrevistas foram realizadas pelos pesquisadores, que são pesquisadores com estudos e publicações nessa temática. Foi utilizado como instrumento de pesquisa, um questionário estruturado composto por oito questões norteadoras que versavam a respeito do conhecimento e aplicação prática do Processo de Enfermagem, elaborado pelos autores, aplicado como parte do protocolo, que incluiu entrevista direta visando à coleta de dados essenciais para determinação dos mesmos. O local de realização das entrevistas foi o ambiente de trabalho do participante, em local privativo e escolhido pelo mesmo.

Todos os pesquisados foram esclarecidos sobre os objetivos e procedimentos do estudo, sendo garantida sua 
participação anônima e na condição de voluntário. Aqueles que aceitaram a participar assinaram o Termo de Consentimento Livre Esclarecido, atendendo à Resolução 466/12 do Conselho Nacional de Saúde.

O projeto de pesquisa foi encaminhado e submetido ao Comitê de Ética em Pesquisa com Seres Humanos, indicado pela Plataforma Brasil, e foi aprovado em 18 de outubro de 2018 com Certificado de Apresentação para Apreciação Ética - $\mathrm{n}^{\circ}$ CAAE 98576818.5.0000.8547 sob o parecer de nº 2.969.613.

\section{Resultados e Discussão}

O estudo abordou diversos eixos relacionados ao desenvolvimento das atividades profissionais enfermeiros que atuam na atenção terciária, ligados diretamente à assistência ao paciente, entretanto elencamos os eixos que estão mais próximos do tema central e, portanto, demonstram claramente sua ligação aos objetivos desse trabalho.

Nesta pesquisa, os participantes totalizaram 18 (100\%) enfermeiros, sendo que 17 (94\%) eram mulheres e um (6\%) era homem, atuantes em unidade de atendimento à saúde em atenção terciária. Observando que a ainda nos dias de hoje a enfermagem é exercida por um maior contingente feminino.

A feminização da enfermagem é um fato histórico que sempre esteve associada à figura matriarcal, devido os serviços que eram realizados nas instituições sacras, sendo essa responsável pelo cuidado às crianças aos velhos doentes e serviços domésticos, além das práticas de saúde e curandeira. E, o desafio é valorizar a profissão, mesmo sendo predominantemente feminina; o que, hoje, leva ao baixo reconhecimento da sociedade, por esperarem que pessoas inteligentes se formem em outras carreiras de saúde. (Lopes \& Leal, 2005; Oliveira, 2018; Silva \& Marcolan, 2015).

Para a utilização dos diagnósticos pelos profissionais enfermeiros e suas opiniões sobre os diagnósticos de enfermagem, todos os participantes afirmaram ter conhecimento sobre os diagnósticos de enfermagem e desenvolvem os mesmos em seu trabalho.

As respostas aos questionamentos foram transcritas integralmente:

Em minha opinião, os diagnósticos de enfermagem são de extrema importância no cuidado de enfermagem, pois os mesmos dão o caminho para a prescrição de cuidados, além disso ajudam na dinâmica do trabalho de nós enfermeiros, porém, na minha visão, falta bastante estudos de nós profissionais, pois na maioria das vezes os diagnósticos já estão montados em chek-list e isso tira o raciocínio do enfermeiro (Enfermeira 3).

Se os diagnósticos de enfermagem fossem montados como devem trariam mais benefícios aos pacientes, e a falta de tempo de nós profissionais em realizar uma anamnese e exame físico prejudica na realização final do diagnóstico de enfermagem (Enfermeira 13).

Para existência de um diagnóstico de enfermagem, é essencial que a etapa inicial do Processo de Enfermagem, ou seja, a coleta de dados deve ser bem sucedida, a mesma não pode ser realizada apenas com intuito de impressos ou formulários eletrônicos (Herdman \& Kamitsuru, 2015; Rodrigues, Ferreira, Lopes, Menezes, Rocha, \& Silva, 2020).

Os mesmo autores afirmam que durante as fases de coletas de dados e diagnósticos do processo de enfermagem, os profissionais reúnem dados não apenas do paciente e sim todo o meio no qual esteja envolvido, família, grupo ou comunidade, essas informações coletadas são processadas e em seguidas são organizadas em categorias de conhecimentos, ou seja, diagnósticos de enfermagem.

Neste pensamento, temos que a enfermagem não coleta dados somente para diagnósticos fisiológicos; e, sim de todo o componente holístico. 
Nessa linha de pensamento, a pesquisa questionou os participantes quais teorias de enfermagem utilizam para nortear os cuidados de enfermagem de acordo com a Resolução 358/2009, que dispõe sobre o processo de enfermagem e sistematização da assistência de enfermagem, Além disso, os profissionais enfermeiros responderam quais sistemas de classificação empregam para os diagnósticos de enfermagem.

Tais questionamentos trouxeram novos problemas a serem investigados, dos 18 (100\%) participantes, apenas 4 $(22,22 \%)$ responderam que se pautam na Teoria das Necessidades Humanas Básicas (NHB) de Wanda de Aguiar Horta e utilizam impressos eletrônicos da Taxonomia da Nanda-I. Os demais 14 (77,77\%), não souberam responder.

Algumas falas justificam a falta de domínio diante ao assunto discutido:

Eu acho que teoria que utilizamos aqui é aquela do livro, se não me engano Nanda, agora classificação aqui não usamos por que já tem no sistema (Enfermeira 8).

Olha é muito difícil utilizarmos as teorias aqui, por que falta tempo e eu não tenho ideia o que seja classificação de diagnósticos, eu entro no sistema e lá já tem alguns diagnósticos prontos, na maioria os diagnósticos são risco de infecção (Enfermeira 15).

A escolha de um referencial nós remete ao conhecimento das teorias de enfermagem, exigindo análise e discussões entre a equipe, levando em consideração à empatia pela teoria, bem como sua forma estrutura onde a mesma será aplicada (Hermida \& Araújo, 2006).

Segundo Lima et al (2006) em um estudo realizado concluíram que uma coleta de dados estando sistematizada favorece o levantamento dos problemas e consequentemente facilita na determinação do diagnóstico e no planejamento das intervenções e resultados de enfermagem.

Os autores acima destacam o papel das teorias de enfermagem afirmando que as mesmas, especificamente na teoria NHB de Horta, são de extrema importância na fundamentação de todo processo de enfermagem.

Segundo Alcântara et al (2011) as teorias de enfermagem tem grande relevância neste processo, pois as mesmas dão um referencial teórico ao trabalho prático do profissional de enfermagem, devido tais serem uma descrição de comportamentos e ações do enfermeiro e que proporcionam, assim, a possibilidade da efetiva implementação da Sistematização da Assistência de Enfermagem (SAE).

Nascimento et al. (2008) corroboram que a utilização da Sistematização da Assistência de Enfermagem, enquanto processo organizacional oferecem subsídios para a evolução de novos métodos e metodologias interdisciplinares para humanização do cuidado. Essas metodologias desenvolvem atualmente uma das mais importantes conquistas na assistência de enfermagem, onde os profissionais que estão diretamente ligados a esse processo, devem buscar e atualizar seus conhecimentos na sua área de atuação.

Backes et al (2005) relatam que embora a maioria dos enfermeiros tenha uma percepção diante da SAE, a realidade concreta, como a escolha de referencial teórico, o processo de implementação e uma metodologia adequada, tem sido ainda um grande desafio.

É essencial destacar abordagem interdisciplinar nesse estudo, onde os profissionais enfermeiros responderam sobre abordagem do tema durante a graduação. Dos 18 (100\%) participantes 13 (72\%) afirmaram não terem dito contato com a temática em alguma disciplina durante a graduação, vindo a conhecer os diagnósticos após a formação acadêmica. O restante 5 (28\%) relataram terem estudado o tema durante a graduação.

Durante a construção da História de Enfermagem, é necessário considerar a possibilidade da interdisciplinaridade, uma 
vez que a mesma agrega um amplo conhecimento, trazendo, portanto uma história mais ampla, teoricamente consistente, com diferentes percepções e significados, fazendo com que exista uma maior compreensão da profissão e identidades para os profissionais que nela atuam (Padilha \& Borenstein, 2006).

O caminho metodológico percorrido nesse estudo nos subsidiou para corroborar e enfatizar a importância do conhecimento técnico embasado cientificamente que o profissional enfermeiro deve ter, visto que é esse empoderamento que valorizará toda a prática do cuidado e nesse interim os diagnósticos de enfermagem merecem destaque na medida em que norteiam o profissional para inúmeras possibilidades interventivas permitindo acompanhar a evolução desses pacientes de maneira menos subjetiva e muito mais objetiva em consonância com seu quadro real de saúde.

\section{Considerações Finais}

O cuidado humanizado, que no caso desse estudo dá enfoque aos diagnósticos de enfermagem considerando-o dentre as fases do PE e em sua essência como chave para uma evolução com prognóstico positivo de cada indivíduo que recebe esse cuidado. Contrariamente a tudo que embasa a ações do enfermeiro, essa pesquisa nos mostrou que muitas vezes os diagnósticos de enfermagem não são aplicados de maneira correta e embasada cientificamente.

Em vista disso analisamos que a adesão dos enfermeiros a uma prática profissional dirigida e pautada em uma ciência, bem como seu conhecimento sobre os diagnósticos de enfermagem demonstrou-se falha, ainda existe um abismo entre o conhecimento prático e científico desses profissionais de enfermagem, estando estes, muitas vezes embasado em chek-list eletrônicos e impressos, sem qualquer fundamentação teórica, visto que as teorias de enfermagem e sistema de classificação são conhecidas apenas por $4(22,22 \%)$ dos $18(100 \%)$ dos participantes.

O papel dos órgãos de classe sistema COFEN/COREN resolução 358/2009, se faz necessário, entretanto os dados da pesquisa mostraram que poucos profissionais receberam uma visita fiscalizatória, e abordagem durante a graduação sobre os diagnósticos de enfermagem foi de apenas $28 \%$ dos entrevistados. Sendo assim, faz se necessário ações que contribuam para capacitação desses profissionais através da inserção de programas de educação continuada e permanente, contribuindo, portanto, para melhora da assistência.

Todo o exposto nos leva a concluir que essa linha de pesquisa merece maior empenho no sentido de se detectar e entender todos os problemas que estão ligados ao PE e SAE, considerando que se trata do principal instrumento de trabalho do profissional enfermeiro e assim necessitam-se do envolvimento de enfermeiros pesquisadores, educadores e gestores em prol da produção de subsídios para os enfermeiros que atuam diretamente na assistência ao indivíduo, família e coletividade.

\section{Referências}

Alcântara, M. R., Guedes-Silva, D., Freiberger, M. F., \& Coelho, M. P. P. M. (2011). Teorias de enfermagem: a importância para a implementação da sistematização da assistência de enfermagem. Revista Científica Da Faculdade De Educação E Meio Ambiente, $2(2), \quad 115-132$. https://doi.org/10.31072/rcf.v2i2.99

Backes, D. S., Esperança, M. P., Amaro, A. M., Campos, I. E. F., Cunha, A. D. O. da, \& Schwartz, E. (2008). Sistematização da assistência de enfermagem: percepção dos enfermeiros de um hospital filantrópico. Acta Scientiarum. Health Sciences, 27(1), 25-29. https://doi.org/10.4025/actascihealthsci.v27i1.1433

Belfort, L. R. M., Sousa, L. T. L., Alencar, A. A. C., Souza, F. E. X., Pereira, A. R. V. L., Santos, M. C., \& Melo, D. E. B. (2020). Sistematização da assistência de enfermagem no processo de gestar: uma revisão integrativa da literatura. Research, Society and Development, 9(8), e816986262. https://doi.org/10.33448/rsdv9i8.6262

Braga, C. G. \& Cruz, D. A. L. M. (2003). A Taxonomia II proposta pela North American Nursing Diagnosis Association (NANDA). Revista Latino-Americana de Enfermagem, 11(2), 240-244. https://doi.org/10.1590/S0104-11692003000200016

Conselho Federal de Enfermagem. (2009, 15 de outubro). Resolução no 358, de 15 de outubro de 2009. Dispõe sobre a Sistematização da Assistência de Enfermagem e a implementação do Processo de Enfermagem em ambientes, públicos ou privados, em que ocorre o cuidado profissional de Enfermagem, e dá outras providências. Diário Oficial da União. http://www.cofen.gov.br/resoluo-cofen-3582009_4384.html

Herdman, T. H. \& Kamitsuru, S. (2015). Diagnósticos de Enfermagem da Nanda. Artmed. 
Research, Society and Development, v. 10, n. 2, e24510212508, 2021

(CC BY 4.0) | ISSN 2525-3409 | DOI: http://dx.doi.org/10.33448/rsd-v10i2.12508

Hermida, P. M. V. \& Araújo, I. E. M. (2006). Sistematização da assistência de enfermagem: subsísios para implantação. Revista Brasileira de Enfermagem, 59(5), 675-679. https://dx.doi.org/10.1590/S0034-71672006000500015

Horta, W. (2011). Processo de Enfermagem. Guanabara-Koogan.

Lepak, S. G. (2015). Fundamentos do Diagnóstico de Enfermagem. In: Herdman, T. H. \& Kamitsuru, S. Diagnósticos de Enfermagem da Nanda. Porto Alegre, Artmed.

Lima, L. R., Stival, M. M., Lima, L. R., Oliveira, C. R., \& Chianca, T. C. M. (2009). Proposta de instrumento para coleta de dados de enfermagem em uma Unidade de Terapia Intensiva fundamentado em horta. Revista Eletrônica De Enfermagem, 8(3). https://doi.org/10.5216/ree.v8i3.7073

Lopes, M. J. M., \& Leal, S. M. C. (2005). A feminização persistente na qualificação profissional da enfermagem brasileira. Cadernos Pagu, 24, 105-125. https://dx.doi.org/10.1590/S0104-83332005000100006

Mazzo, M. H. S. N. (2013). Elaboração e validação de instrumento para consulta de enfermagem à puérpera no âmbito da atenção básica (Tese de Doutorado). Universidade Federal do Rio Grande do Norte, Natal.

Morais, G. J., Brasil, T. C., Tolfo, G. R., Costa, A. E. K., \& Lohmann, P. M. (2020). A Sistematização da Assistência de Enfermagem (SAE) a um paciente com obesidade e hipertensão: relato de experiência. Research, Society and Development, 9(9), e962997940. https://doi.org/10.33448/rsd-v9i9.7940

Nascimento, K. C., Backes, D. S., Koerich, M. S., \& Erdmann, A. L. (2008). Sistematização da assistência de enfermagem: vislumbrando um cuidado interativo, complementar e multiprofissional. Revista da Escola de Enfermagem da USP, 42(4), 643-648. https://dx.doi.org/10.1590/S0080-62342008000400005

Oliveira, E. F. S. (2018). Representação social da profissão enfermagem - reconhecimento e notoriedade. Manole.

Padilha, M. I. C. S. \& Borenstein, M. S. (2006). História da Enfermagem: ensino, pesquisa e interdisciplinaridade. Escola Anna Nery, 10(3), 532-538. https://dx.doi.org/10.1590/S1414-81452006000300024

Rodrigues, I. D. C. V., Ferreira, L. B., Lopes, D. C. L., Menezes, H. F. de, Rocha, C. C. T., \& Silva, R. A. R. da. (2020). Simulação realística: aproveitamento e benefícios para o ensino - aprendizagem do raciocínio diagnóstico de enfermagem. Research, Society and Development, 9(7), e553974338. https://doi.org/10.33448/rsd-v9i7.4338

Silva, D. A., \& Marcolan, J. F. (2015). Desemprego e sofrimento psíquico em enfermeiras. Revista Brasileira de Enfermagem, 68(5), 775-782. https://doi.org/10.1590/0034-7167.2015680502i

Silva, D. R. V. P., \& Moreira, K. F. G. (2020). Intervenção de enfermagem na avaliação e tratamento de feridas em uma Estratégia de Saúde da Família. Recuperado de: https://ares.unasus.gov.br/acervo/handle/ARES/14637

Silva, J. B., Costa, J. S., Melo, A. G., \& Lother, A. M. (2020). Eventos adversos e diagnósticos de enfermagem em pacientes submetidos ao transplante de células-tronco hematopoiéticas. Research, Society and Development, 9(7), e728974618. https://doi.org/10.33448/rsd-v9i7.4618 